\title{
ANÁLISIS DE LA LÓGICA COMBINACIONAL PROPUESTA POR ABB SUECIA AL ICE PARA LA PUESTA EN MARCHA DE LA PROTECCIÓN DE DISTANCIA DE LA SERIE REL 670
}

\author{
Jairo Quirós Tortós
}

\begin{abstract}
Resumen
Esta publicación presenta la descripción de la lógica combinacional propuesta por ABB Suecia para las protecciones de distancia de la serie REL 670, las cuales fueron adquiridas por el Instituto Costarricense de Electricidad (ICE) bajo la licitación $\mathrm{N}^{\circ}$. 102-2005.

El estudio se basa en describir cada bloque funcional y su aporte al funcionamiento de la lógica combinacional del relé. Además, se analizan los ajustes más importantes de los bloques funcionales que componen dicha lógica combinacional.De los resultados analizados, se concluye que la lógica combinacional poseía ciertos errores que fueron cambiados y de esta manera adecuar la lógica al Sistema Eléctrico Nacional (SEN). Con estos cambios dichas protecciones pueden ser instaladas en el SEN y operar sin riesgos de poner en peligro la integridad del mismo.
\end{abstract}

Palabras clave: Protección de distancia, ABB, REL 670, lógica combinacional, set de ajustes.

\begin{abstract}
This work describes the combinational logic proposed by ABB (Sweden) for the distance protections REL 670 . These relays were acquired by the Costa Rican Electricity Institute under tender No. 102-2005.

In addition, it describes each functional block and its contribution to the performance of the combinational logic of the relay. . Also, important adjustments of the functional blocks that make up the combinational logic are analyzed.

The study concluded that the combinational logic had some problems that werecorrected in order to adapt the combinational logic to the national power system. With these changes the protections can be installed across the power system without risking the integrity of the network.
\end{abstract}

Keywords: Distance protection, ABB, REL 670, combinational logic, set of settings.

Recibido: 10 de Marzo del 2010 •Aprobado: 15 de Mayo del 2012

\section{INTRODUCCIÓN}

A cerca de las características de operación importantes, de la protección de distancia ABB REL 670, es que sus ajustes se llevan a cabo con valores en secuencia cero y positiva. Además de que pueden venir totalmente "limpios", o sea, hay que programar la lógica combinacional para las funciones a implementar en el relé, lógica que ya viene programada desde fábrica en otros relés adquiridos por el ICE. Otra característica adicional, es que los ajustes de la línea deben realizarse en valores primarios.
De esta manera, el ajuste asegura un mejor análisis y un menor tiempo de procesamiento, ya que los eventos de un sistema de potencia son fenómenos que se describen en secuencia, evitando de este modo tener que transformar las impedancias de fase en impedancias de secuencia para la comparación de las variables reales con las ajustadas.

Para el diseño de la lógica combinacional es necesario contar con el software de la compañía ABB denominado CAP 531, el cual se instala conjuntamente con el software PCM 600, este último es necesario para el ajuste de cada parámetro de los bloques funcionales. 


\section{Protección de distancia}

Antes de realizar la descripción de la lógica combinacional, se procede a realizar una reseña de la operación y funcionamiento de una protección de distancia, considerando además las funciones extras o bien complementarias de la función de impedancia.

La función de impedancia (ANSI 21) se suele utilizar para niveles de tensión superior a los $110 \mathrm{kV}$ (niveles de tensión superiores a este son utilizados para transmisión de potencia, de modo que las impedancias no son afectadas por la conexión y desconexión de cargas). Su modo básico de operación se basa en la medición y evaluación de la impedancia de corto circuito, la cual corresponde al cociente de la tensión de la línea y la corriente que fluye por la misma, que en el caso típico, es proporcional a la distancia de la falla. Esta impedancia medida se compara con el valor ajustado en el relé, de forma tal que si este valor medido es menor que el fijado se confirma que se trata de una falla dentro de la característica del relé, éste opera y envía la señal de disparo al interruptor.

En caso de una falla es de esperar que este valor de impedancia disminuya debido al efecto que produce la misma sobre la línea protegida. Esta disminución ocasiona que los lazos de medición de impedancia entren en las zonas protegidas del diagrama de impedancia, el cual se muestra en la Figura 1.
Debido a errores en la medición de estos parámetros, errores en los transformadores de medición así como en el valor del ajuste de impedancia de la línea, no es recomendable ajustar el alcance del relé al cien por ciento (100\%) de la longitud de la línea, por lo que en muchos casos se emplea un margen de seguridad de diez o quince por ciento $(10 \%-15 \%)$ de la longitud total (Quirós, 2008). Esto quiere decir que la primera zona se ajustará al ochenta y cinco o al noventa por ciento (85\% - 90\%) de la impedancia de primera zona, o sea, de la línea de transmisión a proteger. El resto de la línea se protege con un paso de sobre alcance, y la segunda zona con escalonamiento en tiempos para asegurar selectividad (ICE, 2005).

El tiempo de disparo se suele ajustar a cero milisegundos (0 ms), para la primera zona (ICE, 2005). Sin embargo, con sistema de comunicación entre los extremos de la línea la protección de distancia puede convertirse en una protección con esquema de comparación con selectividad absoluta (ICE, 2005). Esto proporciona disparos rápidos y selectivos en el cien por ciento (100\%) de la línea, similar a una protección diferencial de línea, pero con la ventaja que también es una protección de respaldo remoto para el sistema (ICE, 2005).

Tal y como se mencionó anteriormente, las protecciones de distancia efectúan la medición con base en la relación entre el voltaje y la corriente que reciben de los transformadores de
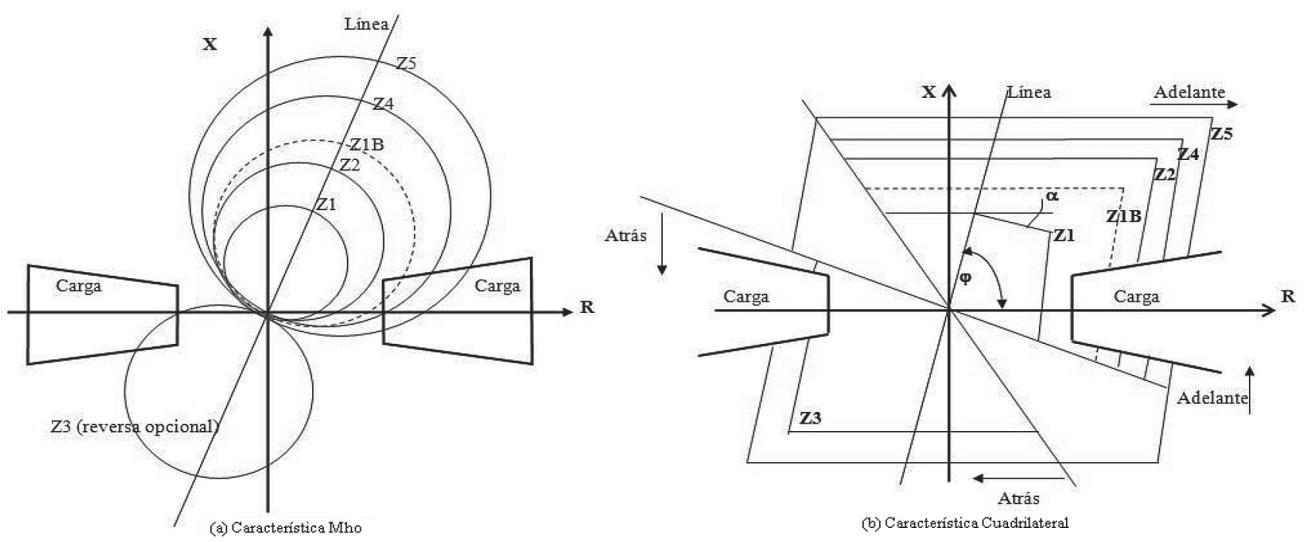

Figura 1. Característica de operación (a) Mho (b) Cuadrilateral.

Fuente: El autor. 
instrumento (transformadores de corriente (TC) y transformadores de tensión (TP)), midiendo por lo tanto valores secundarios de impedancia en unidades de $\mathrm{Ohm}$ secundarios.

Dado que los ajustes del relé REL 670 son en Ohm primarios los valores de los parámetros de pruebas también deben de ser primarios. Además, dado que el ICE ha utilizado valores secundarios en las protecciones adquiridas anteriormente es necesario emplear la Ecuación (1) para determinar los parámetros de ajuste en valores primarios.

$$
Z_{S E C}=\frac{R T C}{R T P} * Z_{P R I M}=\frac{\frac{I_{P R I M}}{I_{S E C}}}{\frac{V_{P R I M}}{V_{S E C}}} * Z_{P R I M}
$$

Donde:

$Z_{\text {PRIM: Impedancia de la línea. }}$

$\mathrm{Z}_{\text {SEC}}$ : Impedancia de la línea reflejada al secundario.

RTC: Relación de transformación del transformador de corriente.

RTP: Relación de transformación del transformador de potencial.

Hoy en día, se suelen obtener protecciones que además de realizar la función de impedancia cuentan con funciones de respaldo contra sobrecorriente (ANSI 67). Estas incluyen además la función de recierre (ANSI 79), la cual es programable (monofásica o trifásica); y en el caso del SEN se ajusta un único recierre ante fallas monofásicas. Otras funciones importantes son la detección de oscilaciones de potencia (ANSI 68) y los esquemas de teleprotección (ANSI 85).

La función de impedancia tiene la opción de operar con dos características diferentes: Mho y la Cuadrilateral (ver Figura 1), siendo la primera muy poco utilizada en el país debido a la poca flexibilidad en el ajuste de los parámetros, dado que su ajuste describe un círculo cuyo diámetro es el valor de la impedancia característica (ICE, 2005). Al no ser homogénea a lo largo de la característica de la línea, si se quiere aumentar el valor de la reactancia se afecta directamente el valor de la resistencia de arco o falla. Por su parte, la característica cuadrilateral es la que se utiliza en el SEN, con ella se tiene la posibilidad de variar independientemente el valor de la parte real e imaginaria de la impedancia, obteniendo así la posibilidad de ajustar cada eje por separado (ICE, 2005).

Estas protecciones de distancia, al igual que otras de su tipo, tienen la capacidad de seleccionar la dirección de la falla, de modo que posee la opción de direccionalidad. Esta operación tiene como objetivo determinar si una falla es hacia adelante (hacia el elemento a proteger) o bien hacia atrás, o dado el caso se encuentra en zona no direccional.

Como se mencionó anteriormente, la función de impedancia determina el valor de la impedancia de falla, la cual es proporcional a la distancia de la línea, de modo que ante una falla posee la función del localizador de fallas la cual tiene como objetivo determinar la distancia aproximada de la falla (Quirós, 2008).

Se observará más adelante que cuenta también con la función de respaldo de sobrecorriente direccional (ANSI 67), esta función debe ser capaz de operar cuando se pierde la señal de tensión.

Otra de las características importantes es la opción de traslapar la impedancia de carga, esto con el objetivo de aumentar el trasiego de potencia, de modo que se disminuye el valor de la resistencia de la línea y así se obtiene una característica operacional ideal mediante la opción de discriminación de carga (load encroachment).

Debidoaerrores en lamedición de los parámetros de las líneas, errores en los transformadores de medida, en el cálculo del valor de impedancia de la línea (ya que está basado en cálculos teóricos y no de medición directa), entre otras, el relé no protege con la primera zona el cien por ciento (100\%) de la línea, es por ello que la implementación de una función que realice dicha protección es necesaria, para ello se cuenta con la teleprotección, la cual tiene como objetivo enviar y recibir señales por parte del relé del otro extremo logrando así una selectividad absoluta de la protección (Quirós, 2008).

Estadísticamente está comprobado que la mayoría de las fallas (50\% aproximadamente) (Billinton \& Norman, 1996) son momentáneas, por lo que se hace necesario contar con una función que restablezca el sistema, esto se logra con la función de recierre (ANSI 79).

Una de las características más comunes en las líneas de transmisión son las oscilaciones de potencia (ANSI 68) las cuales inducen errores de operación de la función de impedancia, es por ello que se cuenta con la función de detección de 
oscilaciones de potencia, la cual bloquea la función de impedancia en caso de que se presente este fenómeno trifásico balanceado (Quirós, 2008).

Otra de las funciones importantes del relé es la capacidad de detectar la pérdida de potencial, la cual opera cuando se detecta la presencia de tensión de secuencia cero y no se detecta corriente de falla a tierra (Quiros, 2008). Esta operación genera una alarma de falla en el circuito de medición de voltaje, o bien pérdida de potencial, la cual puede ser utilizada para bloquear $\mathrm{y} / \mathrm{o}$ desbloquear funciones de protección de acuerdo a los requerimientos de tensión (bloquea la función de impedancia y activa la función de sobrecorriente de respaldo).

Para finalizar, y dejando claro que estas son unas de las tantas funciones con las que cuenta protección de distancia ABB REL 670, se debe mencionar la función supervisora del sincronismo (ANSI 25), la cual no permite el cierre de la línea si ésta detecta una diferencia en amplitud, frecuencia y ángulo de fase respecto a los elementos que se desean interconectar (barra - línea, por ejemplo).

\section{LÓGICA COMBINACIONAL Y AJUSTES DE LA PROTECCIÓN DE DISTANCIA ABB REL 670}

A continuación se describen de manera general los grupos funcionales de la lógica combinacional propuesta por el personal de $\mathrm{ABB}$ Suecia, quienes diseñaron dicha lógica combinacional de manera tal que se ajustara a las necesidades del Sistema Eléctrico Nacional de Costa Rica. Para mayor detalle de dicha lógica combinacional se recomienda la lectura del trabajo realizado por el autor Quirós (2008).

\subsection{Grupo funcional de señales analógicas}

Este grupo funcional tiene como objetivo convertir las señales analógicas, de corriente y tensión, en señales digitales para ser utilizadas por la lógica combinacional del REL 670. Los bloques funcionales utilizados en dicho grupo funcional cuentan con la opción de emitir señales individuales de cada fasor, de corriente o tensión, de igual manera cuenta con la opción de emitir una sola señal que combina los tres fasores en una sola variable. El nombre con el que se identifican estos bloques en la lógica combinacional es SMAI y su configuración se lleva a cabo en la herramienta de matriz de señales.

Entre los ajustes más importantes a realizar para este bloque se encuentra la selección del tipo de medición, ya sea, fase a fase o bien de fase a tierra.

\subsection{Grupo funcional de la función de impedancia}

Este grupo funcional contempla la operación de la lógica de impedancia, detección de oscilaciones de potencia, cierre en falla, localizador de fallas y finalmente la función deslizamiento polar.

La lógica combinacional de algunas de estas funciones cuenta con compuertas lógicas (AND y $\mathrm{OR})$ que son utilizadas para condicionar operaciones o mezclar señales y emitir una única señal.

\section{Función de impedancia}

Con respecto al grupo funcional de la función de impedancia se cuenta con tres bloques funcionales ZDRDIR, FDPSPDIS_21 y ZMQPDIS_21, juntos estos tres bloques llevan a cabo la operación de la función de impedancia, de modo que el primero de estos determina la direccionalidad mediante el ajuste de los ángulos necesarios para determinar la zona hacia adelante (ArgNegRes y ArgDir). En la Figura 2 se muestran los ángulos a ser asignados mediante los ajustes mencionados anteriormente.

En cuanto al segundo bloque mencionado, se debe considerar que posee como característica predefinida la no direccionalidad de las zonas. El objetivo de este bloque es seleccionar los lazos en falla indicando la selección del mismo con direccionalidad, dado que el bloque de direccionalidad envía su señal al de selección de fase. Para realizar esta selección se transponen los tres planos posibles de tipos de fallas (monofásicos, bifásicos y trifásicos), de modo que si el punto de falla en el plano de impedancia toca el plano correspondiente, se indica el arranque de los lazos correspondientes. 
De los ajustes más importantes, y que se detallan en las Figura 3, Figura 4 y Figura 5, son las reactancias de secuencia cero y positiva $(\mathrm{X} 1, \mathrm{X} 0)$, así como las resistencias de falla, tanto fase a fase como fase a tierra (RFFwPP, RFRvPP, RFFwPE, RFRvPE). Es importante mencionar que los ajustes del bloque funcional de selección de fase, deben ser ajustados con valores superiores a los de la zona de mayor alcance, esto para evitar que las zonas ajustadas con característica direccional sean recogidas y se pierda la confiabilidad de la protección.

Además de estos ajustes, se debe considerar la opción de operación de una línea larga y cargada (parámetros que dependen muchode la configuración de la red), para solventar esta necesidad se cuenta con la característica de discriminación de carga (load encroachment). Para ajustar esta propiedad se debe parametrizar las variables RLdFw, RLdRv y ArgLd. En la Figura 6 se muestra cada uno de las variables necesarias para ajustar dicha operación de discriminación de carga.

El tercer bloque funcional mencionado, el cual recopila información de los dos bloques anteriores para determinar la direccionalidad y el lazo en falla, tiene como objetivo la selección de la zona en falla de acuerdo al plano de impedancia diseñado para cada bloque funcional y su respectiva zona. Estos bloques funcionales de cada zona de operación son los encargados de realizar el disparo correspondiente de la zona de impedancia.

Los ajustes más destacados en dichos bloques funcionales son las reactancias y resistencias de secuencia cero y positiva (X0, R0, X1, R1) y las resistencias de falla fase a fase y fase a tierra (RFPP y RFPE). Otro parámetro a considerar es la operación de las zonas de impedancia para fallas entre fases y para fallas monofásicas (OperationPP, OperationPE) así como el tiempo de disparo de cada una de las zonas (tPP y tPE).

Una vez que los tres bloques han operado en el orden explicado, la indicación de la zona en falla, así como la selección del lazo con su respectiva direccionalidad se envían a los bloques respectivos, ya sea para disparo al interruptor, o bien para indicación de eventos.

\section{Detección de oscilación de potencia}

La función de oscilación de potencia es utilizada por la lógica combinacional diseñada para determinar eventos simétricos de este tipo en la red. Cuando se detecta un evento de estos se envía una señal de bloqueo a las zonas

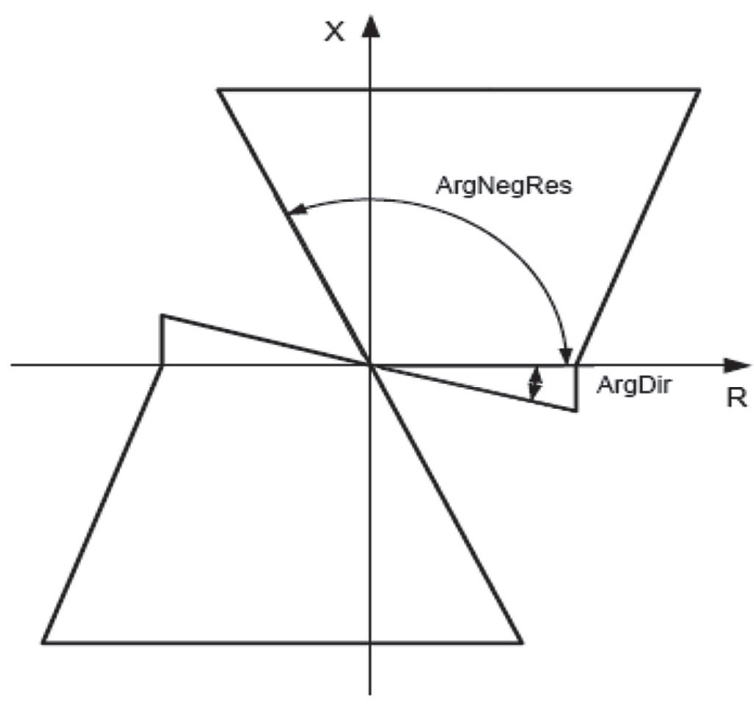

en05000722.vsd

Figura 2. Selección de direccionalidad de la función de impedancia. Fuente: El autor. 


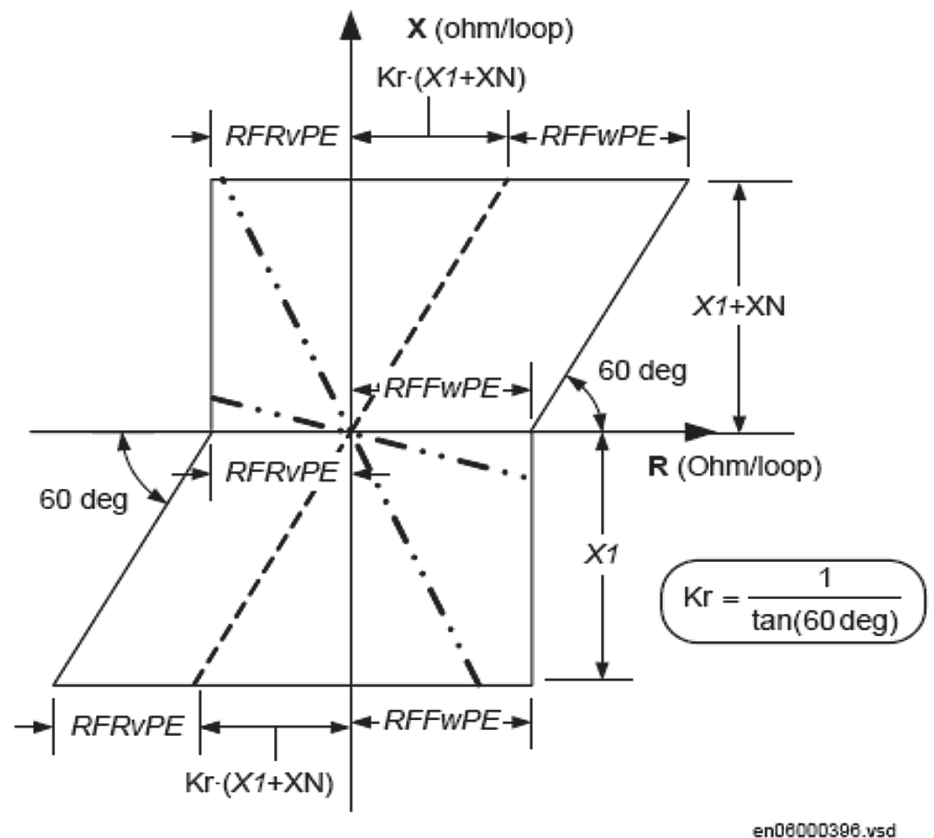

Figura 3. Característica de operación para lazos monofásicos, selección del lazo en falla. Fuente: El autor.

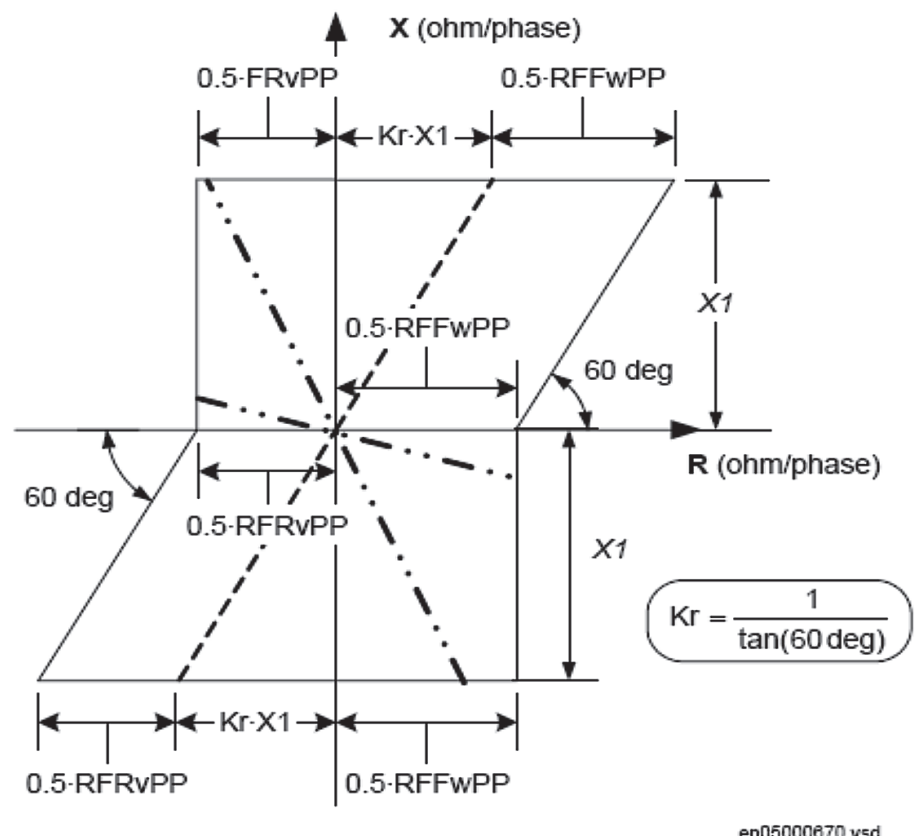

Figura 4. Característica de operación para lazos bifásicos, selección del lazo en falla. Fuente: El autor. 


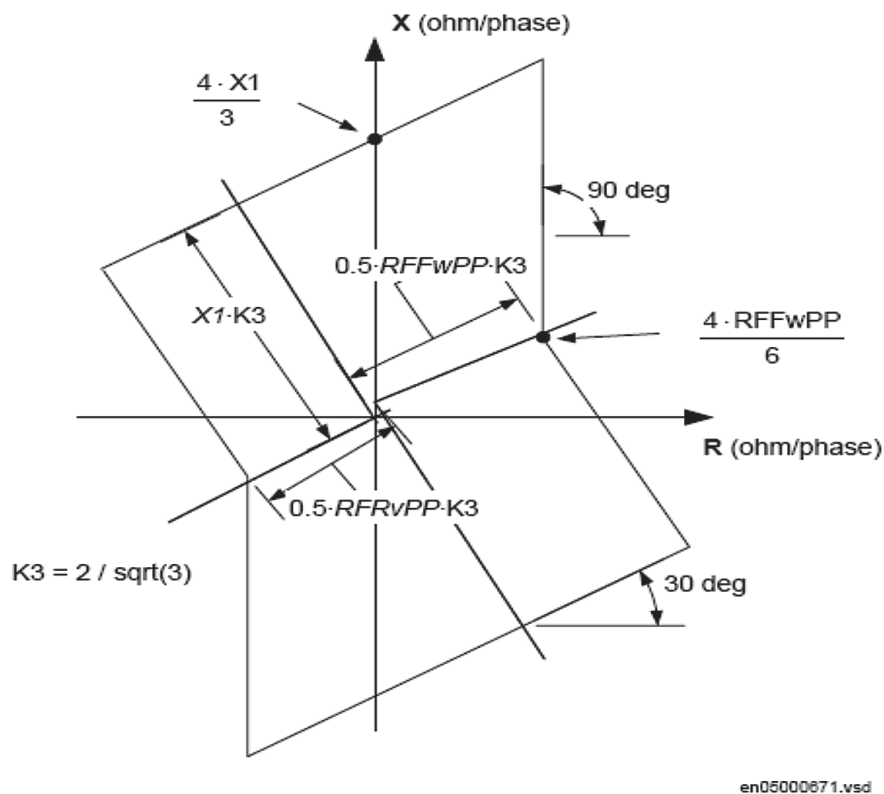

Figura 5. Característica de operación para lazos trifásicos, selección del lazo en falla. Fuente: El autor.

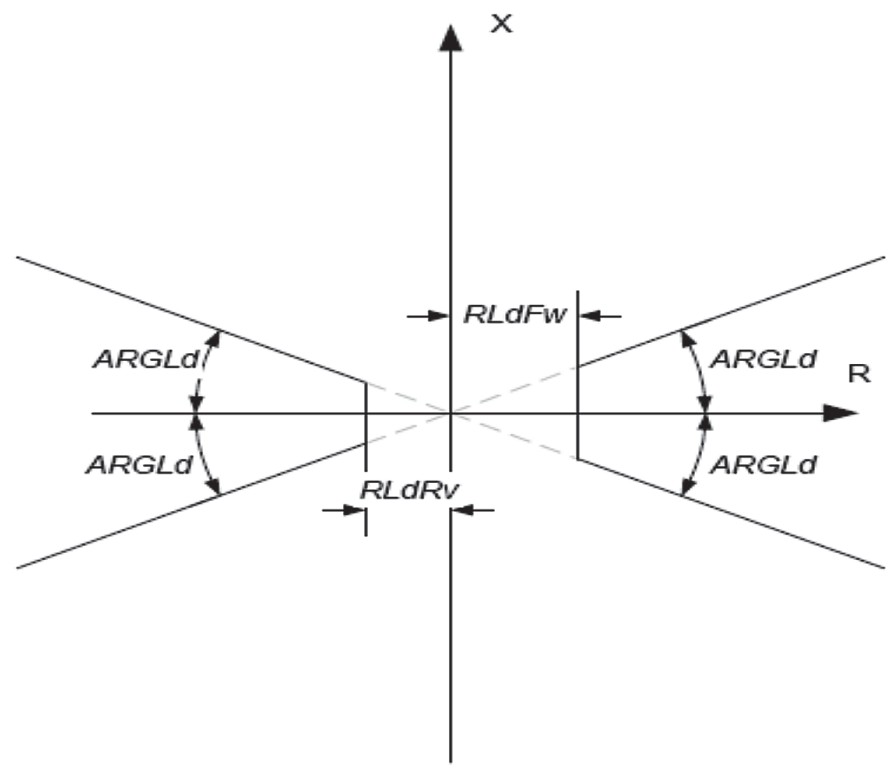

en05000198.vsd

Figura 6. Característica de discriminación de carga, ajustes de dicha operación. Fuente: El autor. 
de impedancia, de modo que no emitan un disparo erróneo. La operación de detección de oscilaciones de potencia es utilizada, además, para enviar disparo a interruptores de modo tal que se aísle el sistema de manera que los flujos de potencia no afecten a toda la red.

En la Figura 7 se muestra los principales ajustes de dicho bloque funcional, dentro de los que destacan los bordes que determinan la razón de cambio de un lazo. Al determinar esta razón de cambio, se compara con la ajustada en los parámetros de tiempo ajustados para dicho bloque funcional. instantánea, esto siempre y cuando se active la compuerta controlable GT03.

Estas compuertas controlables son interruptores lógicos que habilitan o deshabilitan el paso de una señal. Los ajustes de dichas compuertas son: activadas o desactivadas. O sea, permite el paso de la señal o no permite el paso de esta. La función de esta compuerta GT03 en la función de cierre en falla se puede visualizar en la Figura 8. Tal y como se puede observar, la compuerta GT03 es simplemente una compuerta que habilita o deshabilita la función cierre en falla.

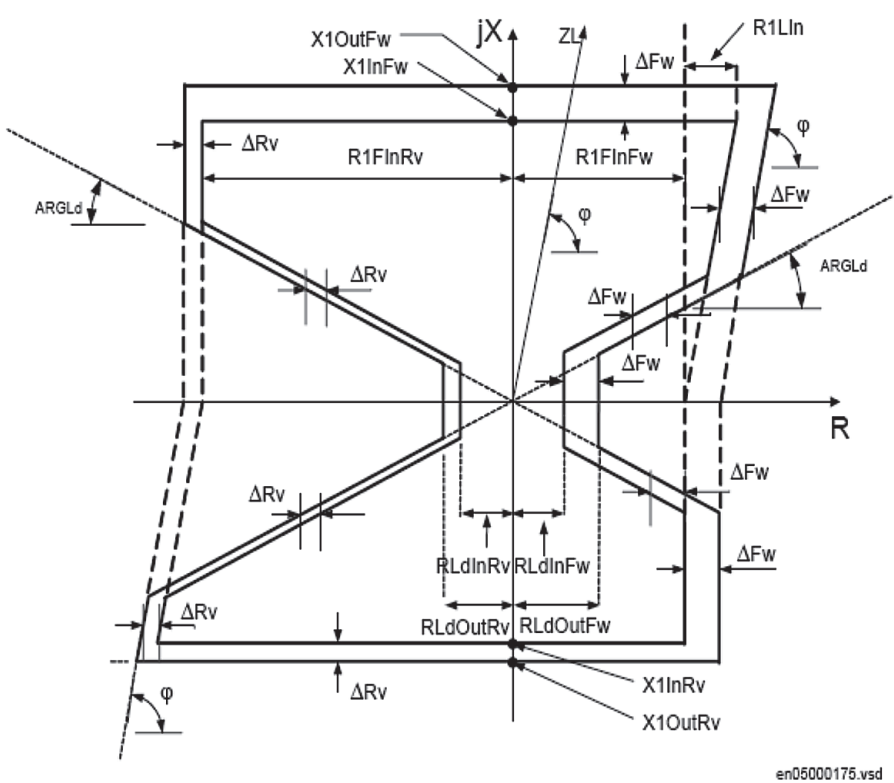

Figura 7. Característica de operación de la detección de oscilación de potencia.

Fuente: El autor.

\section{Función de cierre en falla}

La función de cierre en falla (SOTF) es utilizada para solventar fallas durante el cierre del interruptor de línea, tal y como lo puede ser un error en los enclavamientos de la seccionadora a tierra. La lógica combinacional cuenta con arranque por recierre o bien por comando de cierre manual. Además puede ser acelerado el disparo a partir del arranque de segunda zona o bien por el disparo de la función de sobrecorriente de fase con característica
Tal como se observa en la Figura 9, los bloques funcionales GT cuentan con una única entrada y una única salida. La entrada es una señal digital (por ejemplo disparo al interruptor en la Figura 8) y su salida representa la misma señal habilitada o bien un "cero" digital cuando la compuerta se encuentra desactivada. Estas compuertas tienen un comportamiento similar al de un interruptor en sistemas de potencia, el cual permite el paso de la corriente eléctrica cuando el interruptor está cerrado o bien no permite el paso de dicha corriente eléctrica cuando está abierto. 
Los ajustes más importantes del bloque funcional ZCVPSOF son los umbrales de detección de falla, los cuales son también utilizados para detectar condición de línea muerta. Otros de los ajustes son el tiempo durante el cual se sostiene la señal de disparo (tDuration), el tiempo durante el cual la función permanece activa (tSOTF) y finalmente el tiempo durante el cual se debe cumplir las condiciones de línea muerta para cumplir con esta característica (tDLD).

\section{Localizador de fallas}

El localizador de fallas (FL) es una herramienta que facilita y agiliza las maniobras de mantenimiento. Esto debido a que permite la pronta ubicación del punto de falla en el trayecto de la línea de transmisión. El bloque funcional LMBRFLO es utilizado para realizar dicha operación y las señales emitidas son enviadas directamente al registro del evento, en donde se

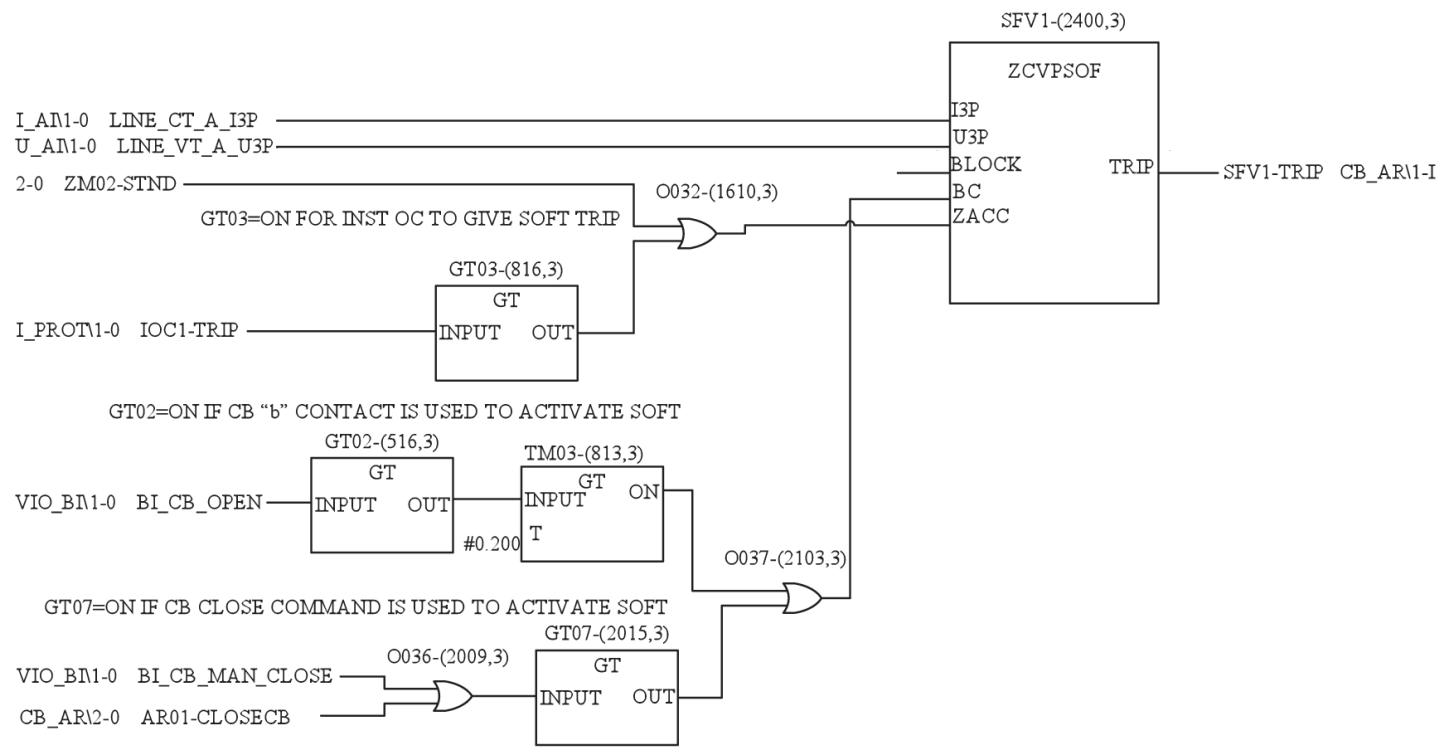

Figura 8. Lógica adicional a la función SOTF.

Fuente: El autor.

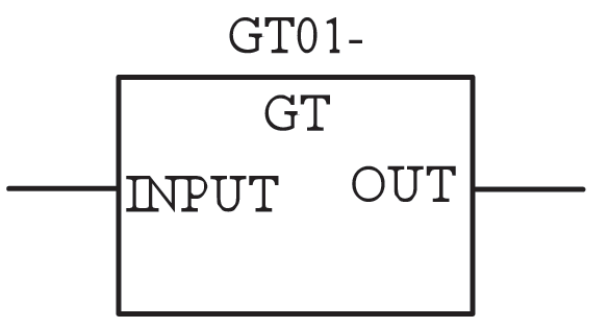

Figura 9. Bloque funcional GT.

Fuente: El autor. 
indica la distancia en porcentaje y en unidades métricas de distancia ( $\mathrm{km}$, millas) dependiendo de la configuración del software instalado en el relé.

Los ajustes más importantes de dicho bloque funcional son las impedancias de secuencia cero y positiva de la línea de transmisión, así como la impedancia equivalente de cada una de las subestaciones a la cual interconecta ducha línea.

\section{Función de deslizamiento del polo}

La función de deslizamiento polar es generalmente utilizada en los generadores, donde la diferencia angular del rotor con el estator provoca la pérdida de estabilidad.

Dado que las protecciones de distancia adquiridas por el ICE son para utilizar en líneas de transmisión, dicha función no es necesaria.

\subsection{Grupo funcional de la función de corriente en reversa y WEI, función de teleprotección}

Este segundo grupo funcional se utiliza para las funciones de conexión a un extremo débil y sobrecorriente en zona inversa, así como para la función de teleprotección, utilizada para aumentar la selectividad de la función de impedancia.

Ambas lógicas combinacionales cuentan con compuertas lógicas que permiten el envió y recepción mediante tres canales independientes de comunicación o bien por un único canal, lo anterior se realiza con la compuerta controlable GT01.

\section{Sobrecorriente en zona inversa y conexión a un extremo débil}

La lógica de la función WEI es utilizada para barras donde la carga es muy baja y las corrientes de cortocircuito son similares a las corrientes de carga. Su implementación se lleva a cabo en la subestación opuesta a la barra débil (en la barra fuerte), y se basa en la operación de la primera zona de operación. Por lo que el arranque de la segunda y quinta zona son utilizadas para bloquear el envío de la señal de disparo al extremo opuesto.
La detección de sobrecorriente en zona inversa, es utilizada para bloqueo de la función de teleprotección explicada a continuación. Esta función basa su operación en el arranque de la quinta zona de impedancia.

\section{Función de teleprotección}

Esta función es de suma importancia para aumentar la selectividad de la función de impedancia. De acuerdo a lo mencionado en la introducción, los transformadores de potencial y de corriente introducen errores que afectan el alcance del 100\% de la línea. Para lograr una total cobertura de la línea se implementa la función de teleprotección, esto de acuerdo a los requerimientos del sistema y al esquema de teleprotección seleccionado. Estos esquemas de teleprotección son: permisivo de sobrealcance, permisivo de subalcance, bloqueo y desbloqueo (Quirós, 2008).

\subsection{Grupo funcional de las funciones de sobrecorriente}

El grupo funcional de sobrecorriente contempla funciones de operación variada. Las funciones con las que cuenta la lógica son: sobrecorriente de fase con característica instantánea, sobrecorriente a tierra con característica instantánea, sobrecorriente de fase con característica inversa, sobrecorriente a tierra con característica inversa, protección térmica de sobrecarga y supervisión de ruptura de conductor.

Inicialmente las protecciones de distancia deben utilizar las funciones de sobrecorriente como funciones de emergencia, sin embargo, se determinó errores de programación, dentro de las que destaca la activación paralela de las funciones de impedancia y sobrecorriente conjuntamente.

De acuerdo a la solicitud y uso de las protecciones de distancia en el Instituto, la función de impedancia debe operar ante la presencia de tensión y la función de sobrecorriente debe operar ante la pérdida de tensión. Para lograr la operación de estas funciones, es necesario contar con la función de falla de fusible, la cual se analizará más adelante. Al analizar la lógica se pudo determinar un error de programación, el cual consiste a que 
ambas funciones operan de manera paralela. En otras palabras, no se programó la función de emergencia de manera correcta.

Las función de sobrecorriente instantánea, de acuerdo a las solicitudes del ICE, serán utilizadas para provocar el arranque de la función cierre en falla.Posteriormente, las funciones con característica inversa serán utilizadas como emergencia.

Los principales ajustes de las funciones de sobrecorriente, son: valores de arranque y disparo del umbral de corriente, tiempo para operación de la función, en caso de ser inverso y por supuesto el ajuste de la característica inversa, ya sea muy inversa, normalmente inversa y moderadamente inversa.

\subsection{Grupo funcional de la función WEI y teleprotección para sobrecorrientes a tierra}

De acuerdo a los requerimientos del SEN y a las solicitudes del ICE, la función de teleprotección es utilizada para aumentar la selectividad de la función de impedancia. Por ello, esta función no será utilizada por el Instituto en el SEN. La aplicación de estas funciones es similar a la mencionada para el grupo funcional explicada en la sección 2.3 .

\subsection{Grupo funcional de las funciones de tensión}

Este grupo funcional cuenta con cuatro principales funciones a saber: falla de fusible, sobretensión, subtensión y pérdida de tensión.

Actualmente el ICE no hace uso de las funciones de tensión como protección, únicamente se utiliza la función falla de fusible, esto para activar o desactivar las funciones de impedancia y sobrecorriente de emergencia.

La operación de estas funciones se basa en la medición de la tensión de línea, con ésta, determina los valores de secuencia, tanto cero como negativa, para comprobar el desbalance en la línea y así comparar con los valores ajustados en el PCM 600.

Con respecto a la función de falla de fusible, ésta es utilizada para determinar el disparo del térmico de potencial, el cual a su vez envía la indicación a las funciones de impedancia y de sobrecorriente de emergencia (sobrecorriente a tierra con característica inversa).

Inicialmente la lógica cuenta con un error de programación en cuanto a las funciones de impedancia y sobrecorriente de emergencia, ya que ambas operan de manera conjunta cuando deberían de operar de manera complementaria.

La determinación del disparo del térmico de potencial se puede efectuar a partir de dos principios básicos. El primero consiste en una entrada binaria, la cual indica, a partir de un contacto externo al relé, la operación del térmico de potencial. El segundo, consiste en la medición de las componentes de secuencia, cero y negativa, para determinar el desbalance de las fases y comparar con los valores ajustados para la operación de la función.

\subsection{Grupo funcional de la función de disparo al interruptor}

El grupo funcional de disparo al interruptor consiste en dos operaciones relacionadas con el operar del interruptor. Principalmente se destacan: disparo al interruptor y determinación de falla de canal de disparo.

En cuanto a la primera operación, consiste en tres bloques funcionales. Los primeros dos bloques, que son iguales, llevan a cabo la recolección de las señales, las que van a disparar trifásicamente, así como las que van a disparar cada fase individualmente.

Posteriormente, se cuenta con una lógica que determina el estado del canal de disparo, esta lógica es utilizada como alarma para comprobar el estado correcto del cable que se envía para disparo. Para activar esta operación, es necesario activar la GT21.

\subsection{Grupo funcional de las funciones de recierre y sincronismo}

En este grupo funcional se encuentran las funciones encargadas de realizar el recierre (ANSI 79), así como la supervisión de sincronización (ANSI 85).

Inicialmente, la función de recierre cuenta con un bloque de preprocesamiento, el cual se encarga de agrupar en una compuerta lógica 
(OR) todas las señales que permiten el arranque de un ciclo de recierre, en este mismo bloque se cuenta con un otra compuerta lógica que agrupa las señales que bloquean el recierre de la línea.

Se debe recordar que el recierre es utilizado como medida de restablecimiento de la línea ante fallas monofásicas, esto en el caso de Costa Rica. Sin embargo, el bloque funcional de recierre cuenta con la opción de realizar hasta cinco ciclos de recierre y con diferentes operaciones para el primer ciclo, mientras que los restantes ciclos son únicamente recierrestripolares debido al disparo tripolar.

Los principales ajustes del bloque funcional de recierre son: número de ciclos de recierre y tiempo de cada ciclo.

En cuanto a bloque de supervisión de sincronismo, el cual funciona también para sincronización de máquinas y supervisión de energización de línea, cuenta únicamente con un bloque funcional que se basa en comparación. El principio de operación de estas funciones, es mediante la comparación de los valores de diferencia de la línea respecto a barra. Estos valores son comparados con los ajustados en el PCM 600 y en caso de que los valores medidos se encuentren dentro de los ajustes de tensión, ángulo de fase y frecuencia, se envía indicación de cierre sincronizado. Para habilitar esta función, es necesario el uso de las compuertas GT11 y GT12, tal como se muestra en la Figura 10. Estas compuertas, las cuales presentaron errores de programación que fueron posteriormente modificados, son necesarias para esquemas de doble interruptor e interruptor y medio. Cuando GT11 está en On se considera únicamente los contactos de posición cerrado de las barras A y B. Si la compuerta GT11 se encuentra en Off la posición de los contactos de abierto de los interruptores de las barras A y B son considerados. Cuando la compuerta GT12 se desactiva (Off) la posición de interruptor cerrado, de las barras A y B es considerado en la lógica, mientras que si esta se activa $(\mathrm{On})$, la posición de cerrado del interruptor no es necesaria, debido a que la lógica siempre considera que se encuentran cerrados.

\subsection{Grupo funcional de las funciones falla de interruptor y discrepancia de fases}

$\mathrm{Al}$ analizar las funciones utilizadas para supervisar fallas en el interruptor se encuentra con las funciones ANSI 50BF y la ANSI 52PD. La primera función se activa una vez ejecutado un disparo, si transcurrido cierto tiempo t1 no se ha abierto el interruptor se cuenta con la opción de ejecutar un segundo disparo a interruptor, o bien, si transcurrido un tiempo 2 no se ha logrado la apertura se envía un disparo a la barra en la cual la línea está conectada. Como característica importante es que estos tiempos son totalmente diferentes.

En cuanto al bloque funcional de discrepancia de fases, éste basa su operación en la medición de los valores de secuencia, de modo que si en cierto momento se ejecuta un disparo monopolar, pero que no es restablecido al transcurir cierto tiempo, se envía disparo a las demás fases, evitando la operación del sistema desbalanceado.

\subsection{Grupo funcional para la medición de señales analógicas}

Todos los bloques mostrados en este apartado se relacionan con la medición de las variables de potencia. Se cuenta con la posibilidad de monitorear las potencias del sistema, corrientes, tensiones, frecuencia, factor de potencia, entre otras. Estas mediciones son de gran importancia para cuando se realizan pruebas de coordinación entre las protecciones y las unidades de control.

Todas las mediciones pueden ser supervisadas mediante cuatro límites ajustables: límite bajo-bajo, límite bajo, límite alto y límite alto-alto.

\subsection{Grupo funcional para la unificación de señales comunes}

Esta lógica se basa en la implementación de compuertas analógicas OR. Tiene como objetivo agrupar todos los posibles disparos, arranques o distintas categorías por medio de una única señal que combina varias condiciones. 


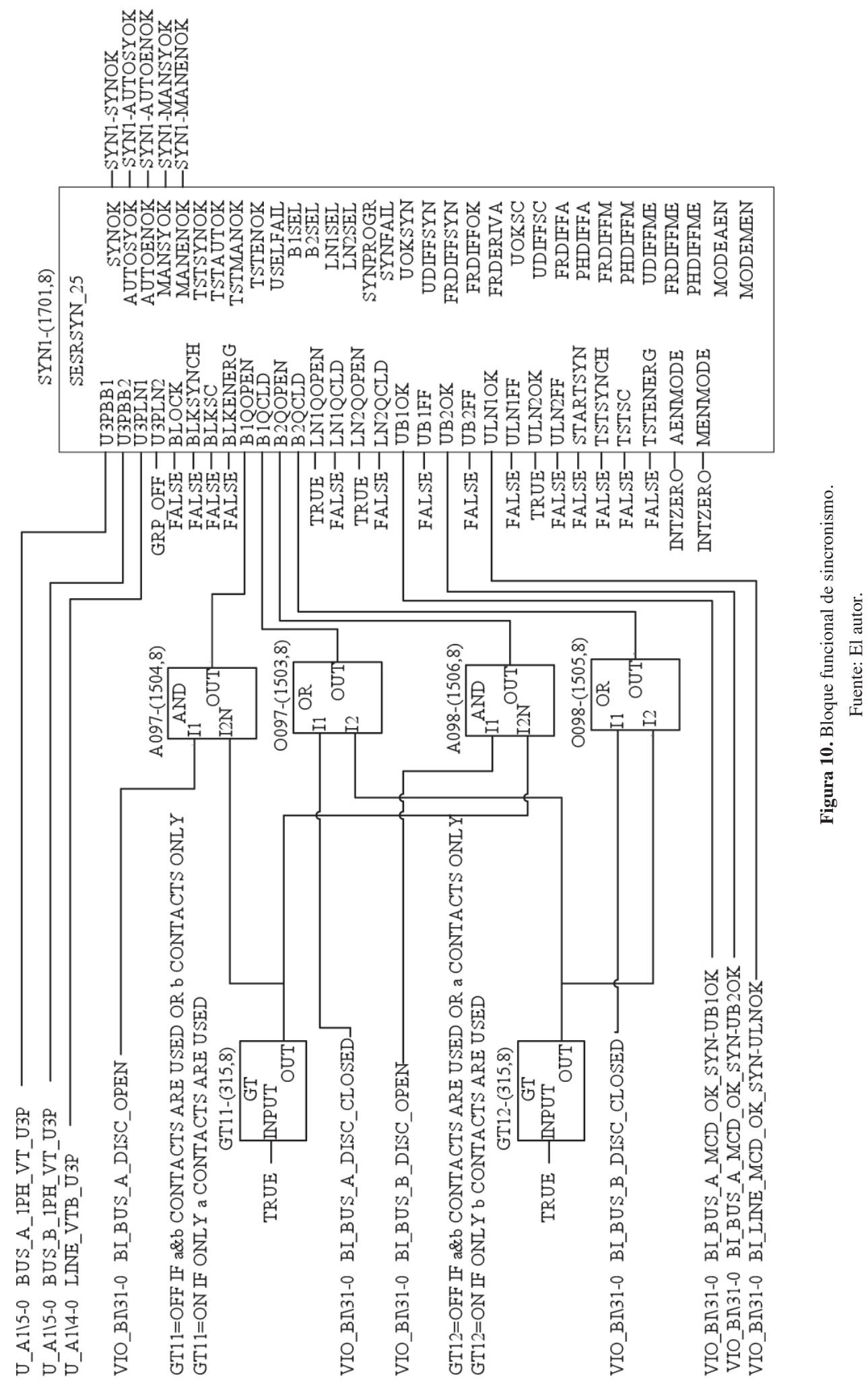




\subsection{Grupo funcional de entradas y salidas binarias}

Estos bloques son utilizados para relacionar los puntos de conexión de las entradas y salidas del hardware del REL 670, con las variables lógicas a ser utilizadas por el software CAP $531 \mathrm{y}$ el PCM 600 mediante la asignación de cada señal con la entrada y salida binaria.

\subsection{Grupo funcional de las señales a registrar en un disturbio}

Este grupo funcional es el encargado de realizar los registros de los eventos. Consiste en una serie de bloques que juntos logran obtener toda la información relacionada con un suceso en la línea.

\subsection{Grupo funcional de señales a ser utilizadas en la lógica combinacional}

En este grupo funcional se ejecutan funciones comunes a todo el relé, tal y como lo son el cambio de opciones de ajuste, definición de variables de estado en la lógica, señales de errores propios del relé y operación de la pantalla del relé y los LEDs.

\section{CONCLUSIONES}

De los resultados obtenidos se desprenden las siguientes conclusiones:

Para una correcta operación de la función de impedancia todos los ajustes correspondientes a los bloques: ZDRDIR (selector de direccionalidad), FDPSPDIS_21 (selector de fase) y ZMQPDIS_21 (zonas de impedancia cuadrilateral) deben estar correctamente parametrizados.

El relé ABB REL 670 cuenta con un set de ajustes adicionales para la selección del lazo en falla, que no existen en otros relés de distancia. Una mala selección de los mismos influye en la rapidez de operación, confiabilidad y selectividad de la protección.

La zona Z5 de impedancia debe ser estrictamente programada con direccionalidad hacia atrás (zona inversa), en caso contrario se tendría una mala operación de los esquemas de bloqueo y POTT.

En relación a la función "cierre en falla", el relé ABB REL 670 cuenta con ajustes adicionales que no están contemplados en otros relés de distancia. Además, existen diferencias significativas en los modos de detección de arranque y condiciones de disparo de este relé en comparación con otros, siendo el relé ABB REL 670 el más complicado en la parametrización, dado que debe contemplar no solamente los modos de operación, sino que además debe haber una concordancia entredichos modos y la entrada programa en la entrada ZACC.

En caso de ser requerido más de un recierre en la línea, la utilización de la señal AR01_CLOSECB tal y como está programada en la función "cierre en falla" podría bloquear la ejecución del ciclo completo de recierres.

Existe un error en la programación del grupo funcional "CB_AR" que impide la ejecución de la función cuando la compuerta GT11 esta desactiva y la compuerta GT12 esta activada.

La programación entregada por ABB Suecia no permite que la función de sobrecorriente trabaje como respaldo de la función de impedancia ante la pérdida de potencial, ni contempla el uso de contactos normalmente cerrados para la activación de la alarma "pérdida de potencial", tal y como lo requiere el Instituto Costarricense de Electricidad.

La función de "falla de interruptor" en el relé ABB REL 670 no contempla el criterio de posición de interruptor para su operación, ni la posibilidad de que los temporizadores de la primera y segunda etapa operen en forma secuencial.

El relé ABB REL 670 posee memoria de almacenamiento de eventos y oscilografías mucho mayor al ochocientos por ciento en comparación con otros relés de distancia, además de que la información desplegada por el mismo es ajustable en la lógica combinacional.

\section{REFERENCIAS BIBLIOGRÁFICAS}

ABB.Application Manual Line DistanceProtection IED REL 670, Código: 1MRK506275UEN_-_TRM_REL670_1.1, Marzo 2007.

ABB. Technical Reference Manual Line Distance Protection IED REL 670, Código: 
1MRK506278-UEN_-_AM_REL670_1.1, Marzo 2007.

Billinton, R. \& Norman, A. (1996). Reliability Evaluation of Power Systems. New York: Plenum Press.

Jonsson, M. (2001). Line Protection and Power System Collapse. Technical Report $\mathrm{N}^{\circ}$ 393L, Department of Electric Power Engineering. Sweden: Chalmers University of Technology.

Lewis B. \& L, Domin J. T. (2006). Protective

Relaying Principles and Applications.Taylor \& Francis Group.

Quirós, J. (2008). Puesta en marcha de la protección de impedancia ABB REL 670. Proyecto de graduación para el grado de Bachillerato. Escuela de Ingeniería Eléctrica. Universidad de Costa Rica.

Stevenson, W.D. (1996). Power System Analysis. New York: McGraw-Hill.

ICE, Subcomité Sistemas de Protección. (2005)"Norma de Diseño de Sistemas de Protección para Subestaciones y Líneas de Transmisión". San Jose: Instituto Costarricense de Electricidad.

\section{NOMENCLATURA}

ABB Asea-Brown-Boveri, Compañía creadora de las protecciones de distancia REL 670.

ANSI Instituto Nacional Americano de Estándares, ANSI por sus siglas en inglés.

BF Falla de interruptor, BF por sus siglas en inglés.

BIM Módulo de entradas binarias, BIM por sus siglas en inglés.

BOM Módulo de salidas binarias, BOM por sus siglas en inglés.
CAP 531 Herramienta de configuración y programación de la compañía $\mathrm{ABB}$.

CB Circuito del interruptor, $\mathrm{CB}$ por sus siglas en inglés.

CENCE Centro Nacional de Control de Energía.

COMTRADE Formato estándar para exportar los archivos del relé de acuerdo a IEC 60255-24.

FL Localizador de fallas, FL por sus siglas en inglés.

ICE Instituto Costarricense de Electricidad.

IEC $61850 \quad$ Estándar de comunicación para la automatización de subestaciones.

IED Dispositivo de Inteligencia Electrónica, IED por sus siglas en inglés.

PCM 600 Herramienta para configuración de los relés ABB.

POTT Esquema de teleprotección de sobrealcance permisivo, POTT por sus siglas en inglés.

SEN Sistema Eléctrico Nacional.

SMAI Matriz de señales para entradas analógicas, SMAI por sus siglas en inglés.

SOTF Cierre en falla, SOTF por sus siglas en inglés.

TC Transformador de corriente.

TP Transformador de potencial.

WEI Extremo remoto débil, WEI por sus siglas en inglés.

\section{SOBRE EL AUTOR}

\section{Jairo Quirós Tortós}

Profesor Interino. Departamento de Máquinas Eléctricas y Sistemas de Potencia, Escuela de Ingeniería Eléctrica, Universidad de Costa Rica. Su área de interés, son los sistemas de protección en alta tensión.E-mail: jairoquirostortos@ieee.org 
